\title{
QUEUEING ANALYSIS FOR TRAFFIC CONTROL WITH COMBINED CONTROL OF DYNAMIC MMPP ARRIVALS AND TOKEN RATES
}

\author{
DOO IL CHOI \\ Department of ApPlied Mathematics, Halla University, South Korea \\ E-mail address: dichoi@halla.ac.kr
}

\begin{abstract}
We analyze the queueing model for leaky bucket (LB) scheme with dynamic arrivals and token rates. In other words, in our LB scheme the arrivals and token rates are changed according to the buffer occupancy. In telecommunication networks, the LB scheme has been used as a policing function to prevent congestion. By considering bursty and correlated properties of input traffic, the arrivals are assumed to follow a Markov-modulated Poisson process (MMPP). We derive the distribution of system state, and obtain the loss probability and the mean waiting time. The analysis is done by using the embedded Markov chain and supplementary variable method. We also present some numerical examples to show the effect of our proposed model.
\end{abstract}

\section{INTRODUCTION}

In telecommunication networks such as ubiquitous network and B-ISDN, by many reasons such as fast transmission, multiplexing and unintentional misbehavior, the congestion is liable to occur. The leaky bucket (LB) scheme has been known as one of the most promising methods for preventive congestion control and policing functions [1]. The operation of the LB scheme can be described by a buffer and a finite token pool in which tokens are generated at a constant rate. A packet ( or cell ) arriving when there are tokens in the token pool departs immediately, consuming one token. If a packet arrives when there are no tokens, the packet must wait in the buffer until next available token is generated. The tokens generated when the token pool is full are lost.

The various LB schemes have been introduced and analyzed by many researchers [2-11]. Specially, we introduce a few interesting papers among them. For a Poisson arrival process, Sidi et al. [2] analyzed the LB scheme with both the finite and infinite buffers. They obtained the distributions of queue length, the waiting time and the inter-departure time. For a Markovmodulated Poisson process (MMPP) as a nonrenewal arrival process, Kim et al. [3] analyzed the finite buffer LB scheme and derived the loss probability and the waiting time distribution. They showed the effects of the system parameters for varying the ratio of the arrival rates and

Received by the editors January 21 2013; Accepted February 282013.

2000 Mathematics Subject Classification. 60K25, 68M20.

Key words and phrases. queueing analysis, leaky bucket, dynamic arrivals and token rates, MMPPs, thresholds, telecommunication networks. 
the sojourn times of each arrival state for the MMPP. There also have been the analysis of the LB schemes by a slotted system ( i.e. discrete-time analysis ). Ahmadi et al. [4] analyzed the LB scheme with batch arrivals in each slots. The solution method is based on the matrix analytic approach. Sohraby et al. [5] extended the batch arrival process to a finite-state discrete Markovian arrival process with infinite buffer and obtained the queue length distribution. For a Poisson and a discrete-time MMPP arrival process with finite and infinite buffers, Wu et al. [6] analyzed the queue length distribution and obtained the ratio of the variance of the interdeparture times to the variance of the interarrival times.

In order to support traffics with different characteristics ( for example, voice, data and video and so on ) and to satisfy Quality of Service (QoS) of traffics, there have been the analysis of the LB schemes with a dynamic token generation intervals or priority [7-12]. The papers [7-9] analyzed the LB scheme with dynamic token generation intervals. Lee and Un [7] analyzed the LB scheme with on-off data source in which the token generation interval during on-period is somewhat smaller than that of off-period. They analyzed the performance of the LB scheme by using the fluid-flow method. Choi et al. [8,9] analyzed the dynamic rate LB scheme in which the token generation interval alternates according to the buffer occupancy. Considering the bursty and correlated properties of traffic, they assumed the arrival process to be the MMPP in continuous-time [8] and Markov-modulated Bernoulli process (MMBP) in discrete-time [9]. Also, Zeng et al. [10] and Choi et al. [11] analyzed the priority LB scheme to support traffics with different characteristics. They classified traffics into two types, the real-time and nonrealtime, and placed two buffers to accommodate each traffic. Zeng et al. [10] analyzed the LB scheme with Head of Line (HOL) priority, in which the cells of high priority are transmitted first than the low priority cells until there is no high priority cells in buffer. In this LB scheme with HOL priority, the QoS of the high priority is absolutely satisfied, while the QoS of low priority may not be satisfied. To overcome this shortcoming, Choi et al. [11] analyzed the LB scheme with queue-length-threshold (QLT) scheduling policy. The high priority are transmitted preferentially depending on buffer occupancy of low priority. Recently, Choi and Lee[12] analyzed the LB scheme with dynamic arrival rates. They place a threshold $L$ on buffer and control the arrivals.

In this paper, we introduce and analyze the LB scheme with dynamic arrivals and dynamic token rates. The motivation for analyzing this model is as follows: In spite of the possibility of congestion in networks, the more packets ( or cells ) can be generated. This deteriorate the network environment. Finally, if the congestion occurs once, the information in network may be lost or become useless. Thus, the network can't satisfy the requirement for service of users. Therefore, for efficient usage of the network resources and fast congestion resolution, the arrivals and token rates need to be controlled together at each congestion level, that is, according to buffer occupancy. We place thresholds $L_{l}(l=0,1,2, \cdots, R)$ on the buffer in order to indicate congestion level of network. According to whether the buffer occupancy exceeds the threshold $L_{l}$ or not, the arrivals and token rates are controlled. Here, it is assumed the arrivals to be MMPPs. This assumption is justified by the bursty and correlated properties of input traffic. 
Following this section, we give the concrete model description in Section 2. In Section 3, by using the embedded Markov chain and supplementary variable method, we derive the system state distribution at token generation instants and arbitrary time. Finally, the loss probability and the mean waiting time are given by system state distribution at an arbitrary time. We also show that the result without thresholds in our LB scheme coincide with the result of existing LB scheme. In Section 4, the some numerical examples are presented to show the effects of our proposed LB scheme.

\section{MODEL DESCRIPTION}

There is a buffer to accommodate the arriving packets and a token pool to store tokens generated. The arriving packets are queued in buffer with finite capacity $K$ if no tokens are available. The token pool has a finite capacity $M$, so that the newly generated tokens are discarded when the token pool is full. Each token allows a single packet to be transmitted, and the token following a transmission is removed from the token pool. We place the threshold values $L_{l}(l=0,1,2, \cdots, R)$ on the buffer and control the arrival process and token rates according to buffer occupancy (that is, queue length). Let $L$ be the queue length at token generation instant. If $L \geq L_{0}$, the token generation interval is $T_{1}$. Otherwise, the token generation interval is $T_{0}\left(T_{1} \leq T_{0}\right)$. Nevertheless, if the queue length increases, we must regulate the arrival stream. Concretely, if the queue length at token generation instant is less than $L_{1}\left(\geq L_{0}\right)$ (i.e. $\left.L<L_{1}\right)$, the arrival follows an MMPP with representation $\left(Q, \Lambda_{1}\right)$. Else, if the buffer occupancy at token generation instant is greater than or equal to $L_{l-1}$ and less than $L_{l}\left(l=2, \cdots, R, L_{i} \leq L_{j}, i<j\right)$, the arrival process follows the MMPP with representation $\left(Q, \Lambda_{l}\right)$. Here, the matrix $Q$ is the infinitesimal generator matrix of an underlying Markov process $J(t)$ with state space $\{1,2, \cdots, N\}$. And the matrix $\Lambda_{l} \triangleq \operatorname{diag}\left(\lambda_{i}^{l}\right)$ is the arrival rate matrix. The stationary probability vector $\Pi$ of the underlying Markov process $J(t)$ is given by solving the equations:

$$
\Pi Q=\mathbf{0}, \quad \boldsymbol{\Pi} \mathbf{e}=1 .
$$

where $\mathbf{e}$ and $\mathbf{0}$ are vectors of size $N$, and are consisted of all ones and zeros, respectively.

Let $M_{l}(t)$ be the number of arriving packets by $\Lambda_{l}$ during the interval $(0, t]$. Now we define the conditional probabilities

$$
\begin{gathered}
p_{i, j}^{l}(n, t)=\operatorname{Pr}\left\{M_{l}(t)=n, J(t)=j \mid M_{l}(0)=0, J(0)=i\right\}, \\
l=1,2, \cdots, R, \quad 1 \leq i, j \leq N .
\end{gathered}
$$

By Chapman-Kolmogorov's forward equations, we have the differential-difference equations for the matrices $P_{l}(n, t) \triangleq\left(p_{i, j}^{l}(n, t)\right)_{1 \leq i, j \leq N}$ :

$$
\frac{d}{d t} P_{l}(n, t)=P_{l}(n, t)\left(Q-\Lambda_{l}\right)+P_{l}(n-1, t) \Lambda_{l}, \quad l=1,2, \cdots, R,
$$

where $P_{l}(-1, t)$ is the matrix 0 . 
Then, it is easily shown that the matrix $P_{l}(n, t)$ has the probability generating function

$$
\begin{aligned}
\bar{P}_{l}(z, t) & \triangleq \sum_{n=0}^{\infty} P_{l}(n, t) z^{n} \\
& =e^{\left[Q+(z-1) \Lambda_{l}\right] t}, \quad|z| \leq 1, \quad l=1,2, \cdots, R .
\end{aligned}
$$

\section{AnAlysis}

3.1. System state distribution at token generation instants. We consider just the stationary probability distribution of the system state. We first derive the system state distribution at token generation instants. Let $B(n)(T(n))$ be the number of packets (tokens) in buffer (token pool, respectively) just after the $n$th token generation instant. Since the arriving packets wait in buffer only if there is no token, we express the state of buffer and token pool as follows:

$$
S(n) \triangleq B(n)+M-T(n) .
$$

That is, if there are $i$ tokens in token pool $(B(n)=0)$, then $S(n)=M-i$. Also, if there are $i$ packets in buffer $(T(n)=0)$, then $S(n)=M+i$. Finally, the process $\{(S(n), J(n)), n \geq 0\}$ is a 2 -dimensional Markov chain with finite state space $\{(0,0), \cdots(0, N),(1,0), \cdots,(M+$ $K-1, N)\}$. Define the matrix $A_{k}$ and $B_{k}^{l}$ as

$$
A_{k}=P_{1}\left(k, T_{0}\right), \quad B_{k}^{l}=P_{l}\left(k, T_{1}\right) \quad k \geq 0, \quad l=1,2, \cdots, R,
$$

Then, the transition probability matrix $\bar{Q}$ of the Markov chain $\{(S(n), J(n)), n \geq 0\}$ is given by

$$
\begin{aligned}
& \bar{Q}= \\
& \left.\qquad \begin{array}{ccccccccccc}
A_{0}+A_{1} & A_{2} & \ldots & A_{M+L_{0}} & A_{M+L_{0}+1} & \ldots & A_{M+L_{l}} & A_{M+L_{l}+1} & \ldots & A_{M+K-1} & \bar{A}_{M+K} \\
A_{0} & A_{1} & \ldots & A_{M+L_{0}-1} & A_{M+L_{0}} & \ldots & A_{M+L_{l}-1} & A_{M+L_{l}} & \ldots & A_{M+K-2} & \bar{A}_{M+K-1} \\
0 & A_{0} & \ldots & A_{M+L_{0}-2} & A_{M+L_{0}-1} & \ldots & A_{M+L_{l}-2} & A_{M+L_{l}-1} & \ldots & A_{M+K-3} & \bar{A}_{M+K-2} \\
\vdots & \vdots & \ddots & \vdots & \vdots & \ddots & \vdots & \vdots & \ddots & \vdots & \vdots \\
0 & 0 & \ldots & A_{1} & A_{2} & \ldots & A_{L_{l}-L_{0}+1} & A_{L_{l}-L_{0}+2} & \ldots & A_{K-L_{0}} & \bar{A}_{K-L_{0}+1} \\
0 & 0 & \ldots & B_{0}^{1} & B_{1}^{1} & \ldots & B_{L_{l}-L_{0}}^{1} & B_{L_{l}-L_{0}+1}^{1} & \ldots & B_{K-L_{0}-1}^{1} & \bar{B}_{K-L_{0}}^{1} \\
0 & 0 & \ldots & 0 & B_{0}^{1} & \ldots & B_{L_{l}-L_{0}-1}^{1} & B_{L_{l}-L_{0}}^{1} & \ldots & B_{K-L_{0}-2}^{1} & \bar{B}_{K-L_{0}-1}^{1} \\
\vdots & \vdots & \ddots & \vdots & \vdots & \ddots & \vdots & \vdots & \ddots & \vdots & \vdots \\
0 & 0 & \ldots & 0 & 0 & \ldots & B_{1}^{l} & B_{2}^{l} & \ldots & B_{K-L_{l}}^{l} & \bar{B}_{K-L_{l}+1}^{l} \\
0 & 0 & \ldots & 0 & 0 & \ldots & B_{0}^{l+1} & B_{1}^{l+1} & \ldots & B_{K-L_{l}-1}^{l+1} & \bar{B}_{K-L_{l}}^{l+1} \\
0 & 0 & \ldots & 0 & 0 & \ldots & 0 & B_{0}^{l+1} & \ldots & B_{K-L_{l}-2}^{l+1} & \bar{B}_{K-L_{l}-1}^{l+1} \\
\vdots & \vdots & \ddots & \vdots & \vdots & \ddots & \vdots & \vdots & \ddots & \vdots & \vdots \\
0 & 0 & \ldots & 0 & 0 & \ldots & 0 & 0 & \ldots & B_{1}^{R} & \bar{B}_{2}^{R} \\
0 & 0 & \ldots & 0 & 0 & \ldots & 0 & 0 & \ldots & B_{0}^{R} & \bar{B}_{1}^{R}
\end{array}\right)
\end{aligned}
$$

where $\bar{A}_{k}=\sum_{n=k}^{\infty} A_{n}$ and $\bar{B}_{k}^{l}=\sum_{n=k}^{\infty} B_{n}^{l}$. 
Finally, the stationary probability vector $\mathbf{x}$ of the Markov chain $\{(S(n), J(n)), n \geq 0\}$ is given by solving the equations

$$
\mathbf{x} \bar{Q}=\mathbf{x}, \quad \mathbf{x} \mathbf{e}=1 .
$$

3.2. System state distribution at an arbitrary time point. In this section we derive the probability distribution of the system state at an arbitrary time point. Let $\tilde{T}(\hat{T})$ be the elapsed (remaining, respectively) token generation time at an arbitrary time point. Define the stationary probabilities and the vectors

$$
\begin{aligned}
y_{n}(j) & =\lim _{t \rightarrow \infty} \operatorname{Pr}\{S(t)=n, J(t)=j\}, \\
\mathbf{y}_{\mathbf{n}} & =\left(y_{n}(1), y_{n}(2), \cdots, y_{n}(N)\right) .
\end{aligned}
$$

We also introduce the notation:

$$
\xi(t)= \begin{cases}1, & \text { if the token is generated by interval } T_{1} \\ 0, & \text { if the token is generated by interval } T_{0},\end{cases}
$$

Furthermore, we define the joint probability distribution of the system state and the remaining token generation time at an arbitrary time $\tau$ as

$$
\alpha_{r}(n, j, x) d x=\operatorname{Pr}\{S(\tau)=n, \xi(\tau)=r, J(\tau)=j, x<\hat{T} \leq x+d x\}, r=0,1 .
$$

Let $\alpha_{r}^{*}(n, j, s)$ and $\boldsymbol{\alpha}_{\boldsymbol{r}}^{*}(\boldsymbol{n}, \boldsymbol{s})$ be the Laplace transform of $\alpha_{r}(n, j, x)$ and the vector with $\alpha_{r}^{*}(n, j, x)$ as its elements:

$$
\begin{aligned}
\alpha_{r}^{*}(n, j, s) & =\int_{0}^{\infty} e^{-s x} \alpha_{r}(n, j, x) d x, \\
\boldsymbol{\alpha}_{\boldsymbol{r}}^{*}(\boldsymbol{n}, s) & \triangleq\left(\alpha_{r}^{*}(n, 1, s), \alpha_{r}^{*}(n, 2, s), \cdots, \alpha_{r}^{*}(n, N, s)\right) .
\end{aligned}
$$

In order to derive $\alpha_{r}^{*}(n, j, s)(r=0,1)$, we define the conditional joint probability $\beta_{r}\left(n, l, j_{1}, j_{2}, x\right) d x$ as

$$
\begin{aligned}
\beta_{r}\left(n, l, j_{1}, j_{2}, x\right) d x=\operatorname{Pr}\{\xi(\tau) & =r, n \text { arrivals by } \Lambda_{l} \text { during } \tilde{T}, J(\tau)=j_{2}, \\
x & \left.<\hat{T} \leq x+d x \mid J(\bar{\tau})=j_{1}\right\}, \quad n \geq 0, l=1, \cdots, R
\end{aligned}
$$

where $\bar{\tau}$ is the starting time of the token generation interval including time $\tau$.

We also define the Laplace transform $\beta_{r}^{*}\left(n, l, j_{1}, j_{2}, s\right)$ of $\beta_{r}\left(n, l, j_{1}, j_{2}, x\right)$ and the matrix $\beta_{r}^{*}(n, l, s)$ with $\beta_{r}^{*}\left(n, l, j_{1}, j_{2}, s\right)$ as $\left(j_{1}, j_{2}\right)$-element:

$$
\begin{aligned}
& \beta_{r}^{*}\left(n, l, j_{1}, j_{2}, s\right)=\int_{0}^{\infty} e^{-s x} \beta_{r}\left(n, l, j_{1}, j_{2}, x\right) d x, \\
& \beta_{r}^{*}(n, l, s)=\left(\beta_{r}^{*}\left(n, l, j_{1}, j_{2}, s\right)\right)_{1 \leq j_{1}, j_{2} \leq N}, \quad n \geq 0, l=1,2, \cdots, R .
\end{aligned}
$$

Then, the vectors $\boldsymbol{\alpha}_{\boldsymbol{r}}^{*}(\boldsymbol{n}, \boldsymbol{s})(r=0,1)$ satisfy the following equations: 
For $0 \leq n<M+K$

$$
\boldsymbol{\alpha}_{\mathbf{0}}^{*}(\boldsymbol{n}, \boldsymbol{s})=\frac{T_{0}}{E} \sum_{k=0}^{\min \left\{n, M+L_{0}-1\right\}} \mathbf{x}_{\mathbf{k}} \beta_{0}^{*}(n-k, 1, s),
$$

where $E \triangleq \sum_{k=0}^{M} \mathbf{x}_{k}\left\{\left(\Lambda_{1}-Q\right)^{-1}+T_{0}\right\} \mathbf{e}+\sum_{k=M+1}^{M+L_{0}-1} \mathbf{x}_{k} \mathbf{e} T_{0}+\sum_{k=M+L_{0}}^{K-1} \mathbf{x}_{k} \mathbf{e} T_{1}$ is the mean interdeparture time of packets[12].

Let

$$
l^{\prime} \triangleq l \quad \text { if } \quad M+L_{l-1} \leq n<M+L_{l}, \quad l=1,2, \cdots, R .
$$

For $M+L_{0} \leq n<M+K$

$$
\begin{array}{r}
\boldsymbol{\alpha}_{1}^{*}(\boldsymbol{n}, \boldsymbol{s})=\frac{T_{1}}{E}\left[\sum_{l=1}^{l^{\prime}-1} \sum_{k=M+L_{l-1}}^{M+L_{l}-1} \mathbf{x}_{k} \beta_{1}^{*}(n-k, l, s) 1_{\left\{l^{\prime}>1\right\}}\right. \\
\left.+\sum_{k=M+L_{l^{\prime}-1}}^{n} \mathbf{x}_{k} \beta_{1}^{*}\left(n-k, l^{\prime}, s\right)\right] .
\end{array}
$$

By the same method as in [12], $\beta_{r}^{*}(n, l, s)$ is given as follows:

$$
\begin{aligned}
& \beta_{0}^{*}(n, 1, s)=\frac{1}{T_{0}}\left[\sum_{k=0}^{n} A_{k} R_{n-k}^{1}(s)-e^{-s T_{0}} R_{n}^{1}(s)\right], \\
& \beta_{1}^{*}(n, l, s)=\frac{1}{T_{1}}\left[\sum_{k=0}^{n} B_{k}^{l} R_{n-k}^{l}(s)-e^{-s T_{1}} R_{n}^{l}(s)\right], \quad l=1,2, \cdots, R,
\end{aligned}
$$

where $R_{n}^{l}(s)=\left(s I-\Lambda_{l}+Q\right)^{-1}\left\{\Lambda_{l}\left(\Lambda_{l}-s I-Q\right)^{-1}\right\}^{n}$.

Finally, substituting $\beta_{r}^{*}(n, l, s)(r=0,1, l=1,2, \cdots, R)$ into above equations, and putting $s=0$, we obtain the system state distribution $\mathbf{y}_{\mathbf{n}}=\boldsymbol{\alpha}_{\mathbf{0}}^{*}(\boldsymbol{n}, \mathbf{0})+\boldsymbol{\alpha}_{\mathbf{1}}^{*}(\boldsymbol{n}, \mathbf{0})$ :

For $0 \leq n<M+L_{0}$

$$
\begin{aligned}
\mathbf{y}_{\mathbf{n}}=\frac{1}{E}\left[\left\{\sum_{k=0}^{\min \left\{n, M+L_{0}-1\right\}} \mathbf{x}_{k} \sum_{m=0}^{n-k} A_{m}+\sum_{k=M+L_{0}}^{n} \mathbf{x}_{k} \sum_{m=0}^{n-k} B_{m}^{1}\right\}\right. \\
\cdot\left(Q-\Lambda_{1}\right)^{-1}\left\{\Lambda_{1}\left(\Lambda_{1}-Q\right)^{-1}\right\}^{n-k-m} \\
\left.-\sum_{k=0}^{n} \mathbf{x}_{k}\left(Q-\Lambda_{1}\right)^{-1}\left\{\Lambda_{1}\left(\Lambda_{1}-Q\right)^{-1}\right\}^{n-k}\right] .
\end{aligned}
$$


For $M+L_{0} \leq n<M+K$

$$
\begin{aligned}
\mathbf{y}_{\mathbf{n}}=\frac{1}{E}\left[\sum _ { k = 0 } ^ { M + L _ { 0 } - 1 } \mathbf { x } _ { \mathbf { k } } \left[\sum_{m=0}^{n-k} A_{m}\left(Q-\Lambda_{1}\right)^{-1}\left\{\Lambda_{1}\left(\Lambda_{1}-Q\right)^{-1}\right\}^{n-k-m}\right.\right. \\
\left.-\left(Q-\Lambda_{1}\right)^{-1}\left\{\Lambda_{1}\left(\Lambda_{1}-Q\right)^{-1}\right\}^{n-k}\right] \\
+\sum_{l=1}^{l^{\prime}-1} \sum_{k=M+L_{l-1}}^{M+L_{l}-1} \mathbf{x}_{\mathbf{k}}\left[\sum_{m=0}^{n-k} B_{m}^{l}\left(Q-\Lambda_{l}\right)^{-1}\left\{\Lambda_{l}\left(\Lambda_{l}-Q\right)^{-1}\right\}^{n-k-m}\right. \\
+\sum_{k=M+L_{l^{\prime}-1}}^{n} \mathbf{x}_{\mathbf{k}}\left[\sum_{m=0}^{n-k} B_{m}^{l^{\prime}}\left(Q-\Lambda_{l^{\prime}}\right)^{-1}\left\{\Lambda_{l^{\prime}}\left(\Lambda_{l^{\prime}}-Q\right)^{-1}\right\}^{n-k-m}\right. \\
\left.\left.-\left(Q-\Lambda_{l^{\prime}}\right)^{-1}\left\{\Lambda_{l^{\prime}}\left(\Lambda_{l^{\prime}}-Q\right)^{-1}\right\}^{n-k}\right]\right]
\end{aligned}
$$

and

$$
\mathbf{y}_{\mathbf{M}+\mathbf{K}}=\mathbf{\Pi}-\sum_{n=0}^{M+K-1} \mathbf{y}_{\mathbf{n}} .
$$

Thus, by using the stationary system state distribution $\mathbf{y}_{\mathbf{n}}$, we obtain the following:

(a) The packet loss probability $\left(P_{\text {loss }}\right)$ for an arbitrary time point:

$$
P_{\text {loss }}=\frac{\mathbf{y}_{\mathbf{M}+\mathbf{K}} \sum_{l=1}^{R} \Lambda_{l}^{*} \mathbf{e}}{\sum_{k=0}^{M+K} \mathbf{y}_{\mathbf{k}} \sum_{l=1}^{R} \Lambda_{l}^{*} \mathbf{e}} .
$$

where $\Lambda_{l}^{*}=\sum_{k=M}^{M+L_{0}} \mathbf{x}_{k} \mathbf{e} \Lambda_{1} 1_{\{l=1\}}+\sum_{k=M+L_{l-1}}^{M+L_{l}-1} \mathbf{x}_{k} \mathbf{e} \Lambda_{l}$.

(b) The mean queue length $\left(M_{q}\right)$

$$
M_{q}=\sum_{i=M}^{M+K}(i-M) \mathbf{y}_{\mathbf{i}} \mathbf{e}
$$

The effective arrival rate $\lambda^{*}$ is given by $\lambda^{*}=\Pi \sum_{l=1}^{R} \Lambda_{l}^{*}$ e.

(c) By Little's law, we obtain the mean waiting time $(W)$ in the system:

$$
W=\frac{M_{q}}{\lambda^{*}\left(1-P_{\text {loss }}\right)} \text {. }
$$

Remark : If there is no change of arrivals and token rates (i.e., $\Lambda_{l}=\Lambda$ for all $l$, and $T_{0}=$ $T_{1}=T$ ), then the system state distribution at an arbitrary time is given by

$$
\begin{gathered}
\mathbf{y}_{\mathbf{n}}=\frac{1}{T} \sum_{k=0}^{n} \mathbf{x}_{\mathbf{k}}\left[\sum_{l=0}^{n-k} A_{l}(Q-\Lambda)^{-1}\left\{\Lambda(\Lambda-Q)^{-1}\right\}^{n-k-l}\right. \\
\left.-(Q-\Lambda)^{-1}\left\{\Lambda(\Lambda-Q)^{-1}\right\}^{n-k}\right]
\end{gathered}
$$


where $B_{l}^{l}=A_{l}(l=1,2, \cdots, R)$.

This coincides with result of [3].

\section{NUMERICAL EXAMPLES}

In this section, we give some numerical examples to show the effect of our proposed LB scheme. We place two threshold values, $L_{1}$ and $L_{2}$ on the buffer. The loss probability and the mean waiting time of our proposed LB scheme are examined as a function of the effective arrival rate. As arrival process of packets, we use two-state MMPPs with

$$
Q=\left[\begin{array}{cc}
-\sigma_{12} & \sigma_{12} \\
\sigma_{21} & -\sigma_{21}
\end{array}\right], \Lambda_{1}=\left[\begin{array}{cc}
\lambda_{1} & 0 \\
0 & \lambda_{2}
\end{array}\right], \Lambda_{2}=\Lambda_{1} / 2, \Lambda_{3}=\Lambda_{1} / 4
$$

In all numerical examples, we take buffer size $K=10, \sigma_{12}\left(=\sigma_{21}\right)=0.1$ and $\lambda_{2} / \lambda_{1}=6$. We also set the token generation intervals $\left(T_{0}, T_{1}\right)$ equal to 1 (i.e. $L_{0}=0$ ).

We vary the system parameters to show the performance measures such as loss probability and the mean waiting time of our proposed LB scheme.

First, in Figs. 1 and 2, the performance measures such as the loss probability and the mean waiting time are compared for each of the LB scheme without threshold, with one threshold $(L)$ and with two thresholds $\left(L_{1}, L_{2}\right)$. When no threshold value is placed on buffer, the arrivals follow MMPP with $\Lambda_{1}$, when one, MMPPs with $\Lambda_{1}$ and $\Lambda_{2}$, and when two, MMPPs with $\Lambda_{1}, \Lambda_{2}$, and $\Lambda_{3}$. From the figures, we can show that the loss probability and the mean waiting time are improved when the more thresholds are placed on buffer.

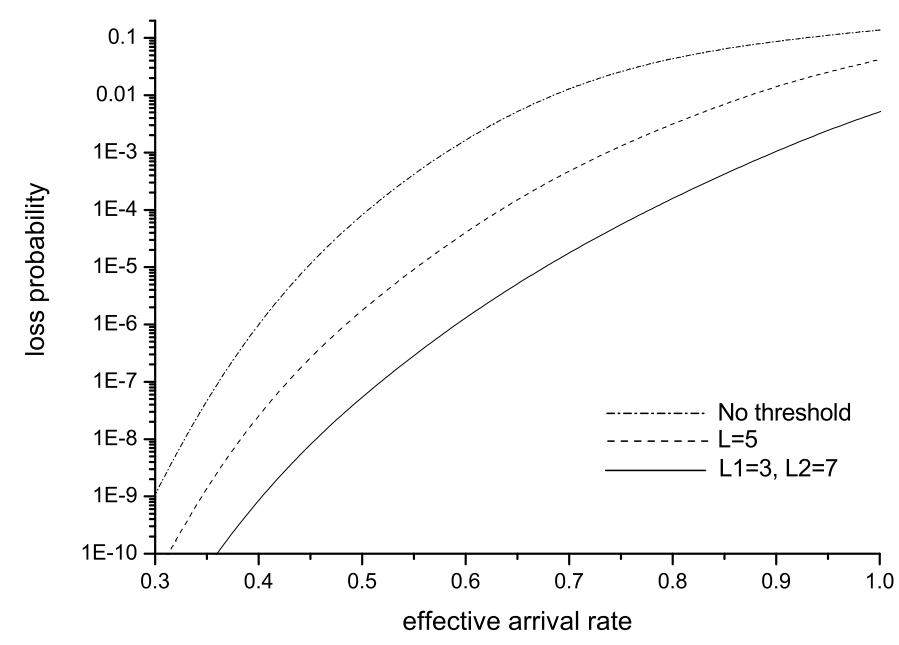

FIGURE 1. Loss probability against total effective arrival rate 


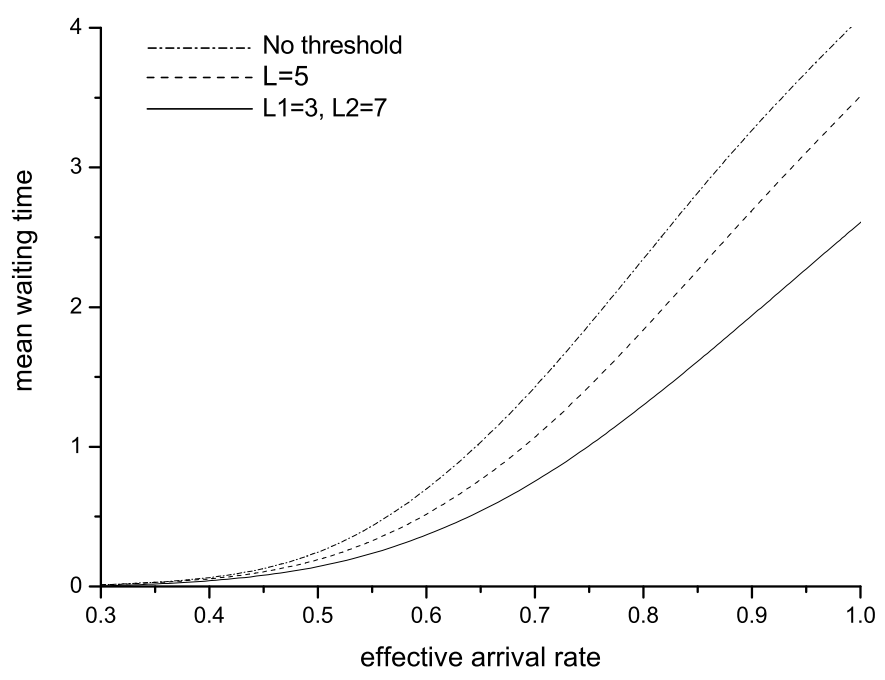

FIGURE 2. Mean waiting time against total effective arrival rate

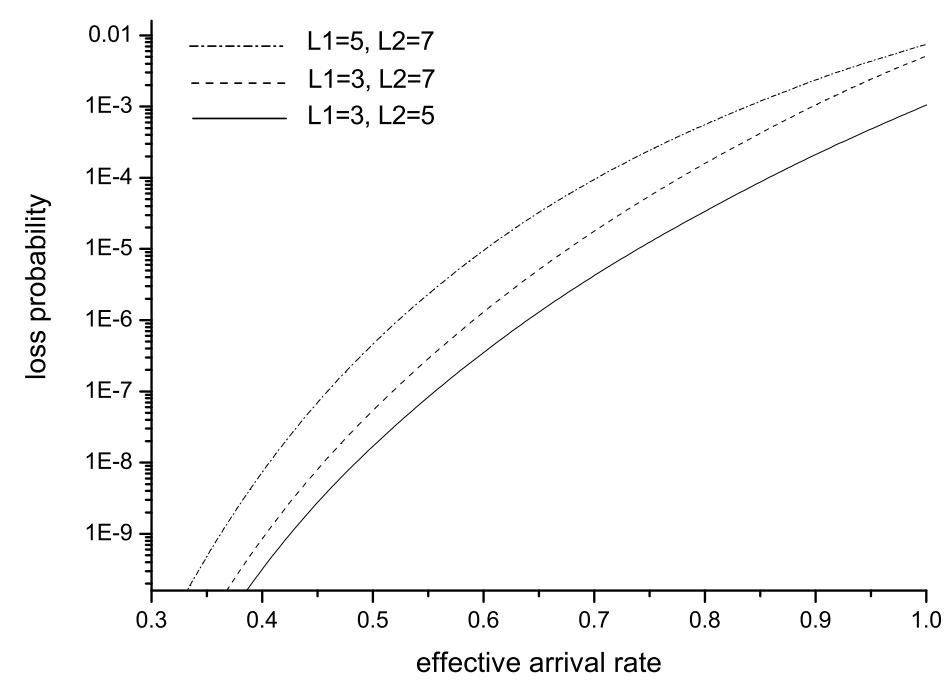

FIGURE 3. Loss probability against total effective arrival rate 
Figs. 3 and 4 illustrate the loss probability and the mean waiting time, respectively, for various values of the threshold values $L_{1}$ and $L_{2}$ when $M=5$. From the figures, we can observe that the loss probability and the mean waiting time are improved when the threshold values $L_{1}$ and $L_{2}$ are given by smaller value relatively.

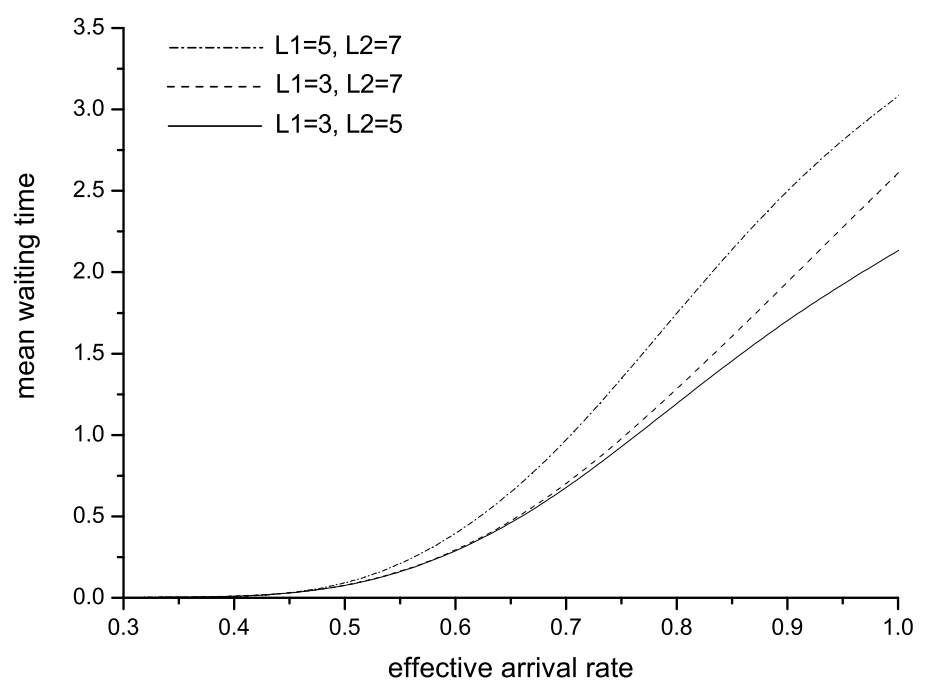

FIGURE 4. Mean waiting time against total effective arrival rate

These results show that by choosing appropriate system parameters such as the number of thresholds, threshold values and token pool size, we can control important performance measures such as loss probability and the waiting time. Finally, with appropriate system parameters, our proposed scheme can satisfy the QoS of users, and prevent congestion which may occur in networks.

\section{CONCLUSIONS}

In this paper we analyzed the queueing model with dynamic MMPP arrivals and token rates. With numerical examples, we examined how various system parameters and arrival processes affected system performance. We expect the analysis results and the numerical examples to help the system designers select appropriate system parameters and arrival process, when they design their systems to prevent congestions and satisfy QoS of users.

\section{REFERENCES}

[1] J. Tunner, New Directions in Communications(or Which Way to the Information Age), IEEE Communications Magazine, 24 (1986), 17-24. 
[2] M. Sidi, Z. Lin, I. Cidon, I. Gopal, Congestion Control through Input Rate Regulation, Proceedings of IEEE Globecom'89 1989.

[3] Y. H. Kim, B. C. Shin, C. K. Un, Performance Analysis of Leaky Bucket Band-width Enforcement Strategy for Bursty Traffics in an ATM Networks, Computer Networks and ISDN System 25 (1992) 295-303.

[4] H. Ahmadi, R. Guerin, K. Sohraby, Analysis of Leaky Bucket Access Control Mechanism with Batch Arrival Process, IEEE Globecom'90 (1990) 344-349.

[5] K. Sohraby, M. Sidi, On the Performance of Bursty and Modulated Sources subject to Leaky Bucket Rate based Access Control Schemes, IEEE Transactions on Communications 42 (1994) 477-487.

[6] G. L. Wu, J. W. Mark, Discrete Time Analysis of Leaky Bucket Congestion Control, Computer Networks and ISDN System 26 (1993) 79-94.

[7] J. Y. Lee, C. K. Un, Performance of Dynamic Rate Leaky Bucket Algorithm, IEE Electronics Letters 29 (17) (1993) 1560-1561.

[8] C. G. Park, B. D. Choi, D. K. Sung, Performance Analysis of Leaky Bucket Scheme with a Threshold in the Data Buffer, Computer Networks and ISDN System 29 (1997) 781-795.

[9] B. D. Choi, D. I. Choi, Discrete-time Analysis of the Leaky Bucket Scheme with Threshold-based Token Generation Intervals, IEE Proceedings of Communications 143 (1996) 105-111.

[10] J. Zeng, L. F. Merakos, Analysis of a Priority Leaky Bucket Regulator for Virtual Path Traffic in ATM Networks, Proceedings of IEEE Globecom'94 (1994) 606-611.

[11] D. I. Choi, B. D. Choi, D. K. Sung, Performance Analysis of Priority Leaky Bucket Scheme with Queue-lengththreshold Scheduling Policy, IEE Proceedings of Communications 145(6) (1998) 395-401.

[12] D. I. Choi, S. Lee, Performance analysis of the leaky bucket scheme with queue length dependent arrival rates, Bulletin of the Korean Mathematical Society 43(3) (2006) 657-669.

[13] D. I. Choi, T. S. Kim, S. Lee, Analysis of an MMPP/G/1/K queue with queue length dependent arrival rates, and its application to preventive congestion control in telecommunication networks, European J. of Operational Research 187 (2008) 652-659. 